\title{
Maternal Hypomethylation of KvDMR in a Monozygotic Male Twin Pair Discordant for Beckwith-Wiedemann Syndrome
}

\author{
S.C. Elalaoui ${ }^{a}$ b $\quad$ I. Garin ${ }^{c}$ A. Sefiani ${ }^{a}{ }^{a}$ b G. Perez de Nanclares ${ }^{c}$ \\ ${ }^{a}$ Département de Génétique Médicale, Institut National d'Hygiène, and ${ }^{b}$ Centre de Génomique Humaine, \\ Faculté de Médecine et de Pharmacie, Université Mohammed V Souissi, Rabat, Morocco; ' ${ }^{\top}$ Molecular (Epi)Genetics \\ Laboratory, Hospital Universitario-Araba-Txagorritxu, Vitoria-Gazteiz, Spain
}

\section{Key Words}

Beckwith-Wiedemann syndrome · Discordant twins .

Methylation defect $\cdot$ Monozygotic twins

\begin{abstract}
Beckwith-Wiedemann syndrome (BWS; OMIM 130650) is a heterogeneous overgrowth syndrome characterized by visceromegaly, macroglossia, tumor predisposition, and other congenital abnormalities. BWS is usually associated with abnormalities of chromosome $11 \mathrm{p} 15$, including (epi)genetic changes, paternal disomy and point mutations. A number of identical twin pairs, mostly female, have been reported to be clinically discordant for BWS. Studies of monozygotic twins discordant for BWS provide more information about failure in the DNA methylation maintenance machinery during very early embryonic development. Here, we report a case of monozygotic male twins discordant for BWS phenotype. Methylation analysis of the 2 imprinted domains at $11 \mathrm{p} 15.5$ (H19DMR and KvDMR) was performed by methylation-specific MLPA and pyrosequencing of DNA extracted from peripheral blood and buccal swabs of both twins. Hypomethylation at KvDMR was identified in both cell types of the affected twin, whereas his healthy brother presented hypomethylation only in blood cells and a normal methylation
\end{abstract}

profile in buccal swab. For diagnostic purposes, it is important to remember that twins can share fetal circulation and possibly share hematopoietic stem cells early in development; therefore, the affected and unaffected twins can share an epigenotype that will resemble partial hypomethylation. If a patient is a twin, it is valuable to obtain a sample from a tissue other than blood.

(c) 2013 S. Karger AG, Basel

Beckwith-Wiedemann syndrome (BWS; OMIM 130650) was first described by Beckwith in 1963 and Wiedemann in 1964 [Beckwith, 1963; Wiedemann, 1964]. BWS is a phenotypically and genetically heterogeneous overgrowth syndrome associated with an increased risk for embryonal tumor development [Pettenati et al., 1986; Elliott et al., 1994; Weng et al., 1995] and occurs with an incidence of 1 in 13,700 live births [Weksberg et al., 2010].

Nearly $85 \%$ of reported BWS cases are sporadic, but $15 \%$ of them are familial. BWS is characterized by macroglossia and gigantism as originally described by Beckwith and Wiedemann, although other clinical characteristics as abnormal growth manifested by hemihyperplasia, and, less frequently, difficulties in feeding and speech and sleep apnea can also be found. Hypoglycemia is re-

\section{KARGER}

E-Mail karger@karger.com

www.karger.com/msy
(C) 2013 S. Karger AG, Basel

1661-8769/13/0051-0041\$38.00/0
Siham Chafai Elalaoui

Département de Génétique Médicale

Institut National d'Hygiène

27 Avenue Ibn Batouta, B.P. 769, 11400 Rabat (Morocco)

E-Mail sihamgen@yahoo.fr 
ported in $30-50 \%$ of babies with BWS [Engstrom et al., 1988]. Children with BWS have an overall risk for tumor development estimated at 7.5\% [Tan and Amor, 2006], with Wilms' tumor and hepatoblastoma being the most common, but other embryonal tumors have also been reported, including rhabdomyosarcoma, adrenocortical carcinoma and neuroblastoma [Lapunzina, 2005]. The BWS phenotype has a wide spectrum, from hemihyperplasia and nevus flammeus to intrauterine, neonatal, or pediatric death [Weksberg et al., 2010]. Final growth parameters are usually within normal limits; however, growth parameters should be followed regularly. The differential diagnosis includes Simpson-Golabi-Behmel syndrome, Sotos syndrome, Costello syndrome, Perlman syndrome, and mucopolysaccharidosis type VI.

BWS is caused by various epigenetic and/or genetic alterations that dysregulate the imprinted genes on chromosome $11 \mathrm{p} 15.5$. The BWS critical region contains 2 domains: the differentially methylated region H19DMR, which regulates the expression of IGF2 and H19 in domain 1; and the differentially methylated region KvDMR, which regulates the expression of CDKN1C, KCNQ1OT1, and KCNQ1 in domain 2. In more than $80 \%$ of individuals with BWS, molecular genetic testing can detect alterations causing BWS. Loss of methylation in KvDMR on the maternal chromosome is present in around $50 \%$ of cases, and gain of methylation in H19DMR on the maternal chromosome is present in around $5 \%$ of cases. Mutations of the maternal CDKN1C allele ( $5 \%$ of cases with no family history, $\sim 40 \%$ of cases with family history) have also been reported. Paternal uniparental disomy of 11p15.5 ( 20\% of cases) and duplication ( $<1 \%$ of cases) or inversion/ translocation $(<1 \%$ of cases) involving $11 \mathrm{p} 15.5$ have been described in BWS patients [Reik and Maher, 1997; Bliek et al., 2001; Cooper et al., 2005; Weksberg et al., 2005, 2010]. However, $20 \%$ of BWS patients have unknown (epi)genetic cause(s). Other genes have been demonstrated to be involved in single families with atypical BWS: the NSD1 gene was found mutated in 2 patients [Baujat et al., 2004]. Also, mutations in NLRP2 at $19 \mathrm{q} 13.42$ have been reported in 2 children of 1 BWS family with multilocus methylation defect [Meyer et al., 2009]. There are some genotype-phenotype correlations; for instance, the epigenetic abnormalities of H19DMR are associated with high risk for tumor development, specifically nephroblastoma [Bliek et al., 2001; Cooper et al., 2005].

An increased frequency of monozygotic (MZ) twinning has been observed in BWS patients [Weksberg et al., 2002; Bliek et al., 2009]. Moreover, twinning in BWS affects almost exclusively females, is nearly exclusively caused by hypomethylation in KvDMR and is mostly associated with discordance, i.e. one twin is affected, while the other is less or unaffected. The remarkable prevalence and discordance of BWS twins indicates that the imprinting failure is at least contemporaneous with twinning and possibly causally connected [Weksberg et al., 2002; Bliek et al., 2009].

Here we report a male MZ twin pair discordant for BWS with their detailed DNA methylation study. DNA methylation data obtained from blood and buccal swab samples of the twins and their parents are presented. We show that the phenotype is mainly due to absence of methylation at maternally imprinted KvDMR.

\section{Patients and Methods}

\section{Clinical Report}

The male twins were delivered at 36 weeks of gestation by caesarean section. At birth, the affected twin weighed 2,200 g, the unaffected twin 2,100 g. The twin pregnancy was the second pregnancy of healthy unrelated Moroccan parents. At birth of the twins, the family consisted of the 35-year-old mother, the 39-yearold father and an 8-year-old healthy brother. At 4 months, the affected twin was $62 \mathrm{~cm}$ in length (50th centile), his weight was 7.2 $\mathrm{kg}$ (75th centile), and his head circumference was $42 \mathrm{~cm}$ (50th centile). He had neonatal hypoglycemia. His appearance was characteristic of BWS with macrosomia, macroglossia, omphalocele, inguinal hernia, neonatal hypoglycemia, and ear creases. The psychomotor development was normal. The unaffected twin had no clinical features of BWS, with normal measurements at 4 months. His length was $58 \mathrm{~cm}$ (10th centile), his weight was $5.6 \mathrm{~kg}$ (10th centile), and his head circumference was $41 \mathrm{~cm}$ (25th centile). The older brother was healthy. The parents had no dysmorphic features of BWS.

At a second clinical exam at age 1 year, the affected twin had a length of $76 \mathrm{~cm}$ (50th centile), a weight of $11 \mathrm{~kg}$ (75th centile) and a head circumference of $47 \mathrm{~cm}$ (75th centile). The unaffected twin was $74 \mathrm{~cm}$ in length (25th centile), his weight was $9 \mathrm{~kg}$ (10th centile), and his head circumference was $46 \mathrm{~cm}$ (50th centile). The twins were regularly monitored for weight and height; furthermore, the BWS-affected twin had abdominal ultrasound and serum alpha fetoprotein level monitoring every 3 months.

\section{Molecular Studies}

Informed consent was obtained from the probands' parents prior to implementation of the genetic studies reported here. Peripheral blood and buccal epithelial cells (buccal swab) were collected from the twins at the age of 1 year (affected and unaffected) and their parents.

Genomic DNA was extracted from blood and buccal swabs using QIAamp DNA Mini Kit (Qiagen, Hilden, Germany) in accordance with the manufacturer's instructions.

Methylation Analysis by Methylation-Specific MLPA

Methylation status of the 2 imprinted domains at $11 \mathrm{p} 15.5$ (H19DMR and KvDMR) was determined by methylation-specific MLPA (MS-MLPA) using the SALSA MLPA kit ME030-B2-BWS/
42

Mol Syndromol 2014;5:41-46 DOI: $10.1159 / 000356689$
Elalaoui/Garin/Sefiani/

Perez de Nanclares 
Fig. 1. MS-MLPA methylation results from blood (a) and buccal swabs (b). c Methylation results from pyrosequencing experiments of $8 \mathrm{CpG}$ islands analyzed at KvDMR in blood and buccal swabs. White bars correspond to the median of 5 independent controls, black bars to the index patient and grey bars to the healthy twin.
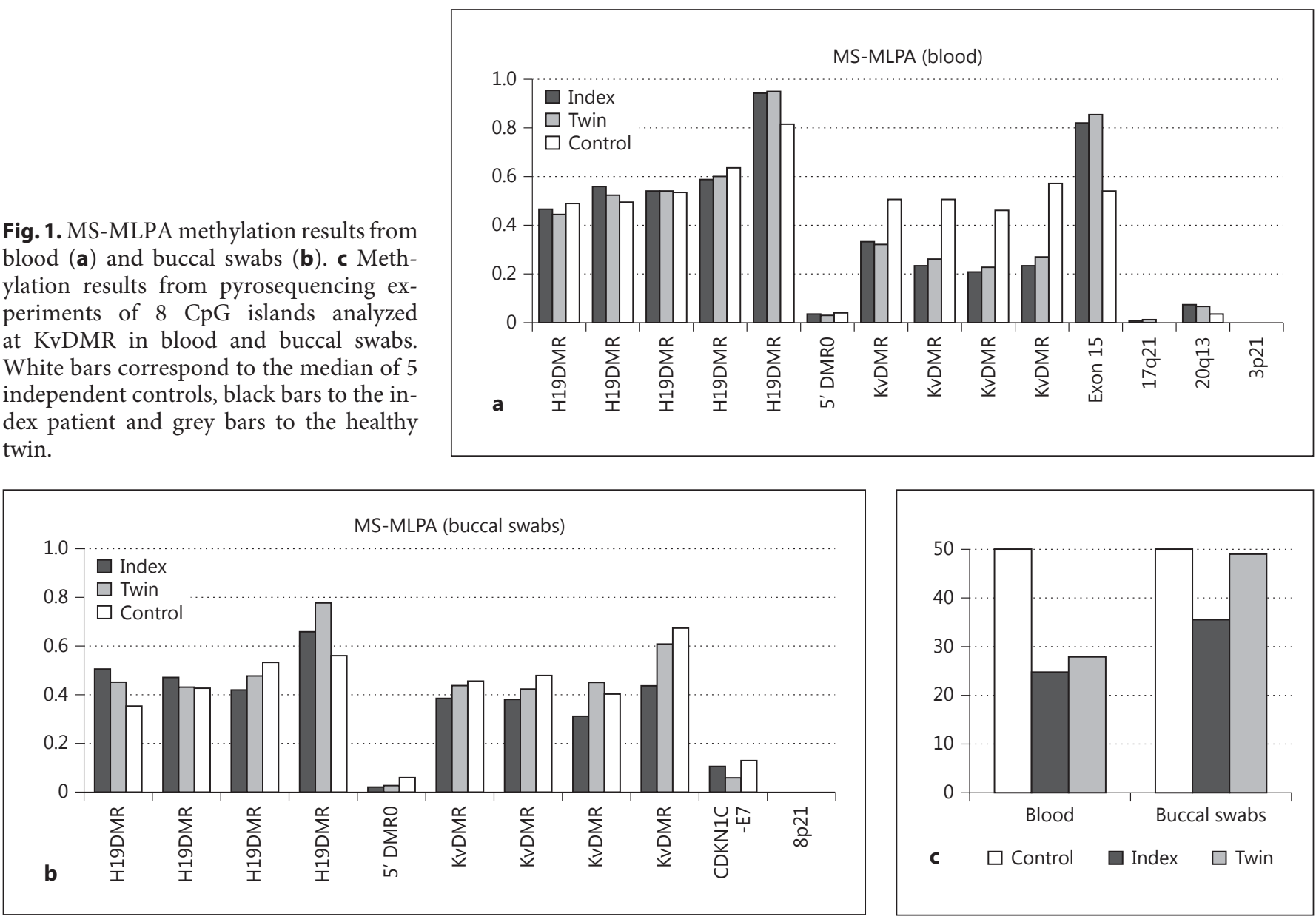

RSS (MRC-Holland, Amsterdam, The Netherlands), in accordance with the manufacturer's instructions. MS-MLPA is a semiquantitative method for methylation profiling. It is a variant of the MLPA technique in which copy number detection is combined with the use of a methylation-sensitive restriction enzyme. Analysis of the MS-MLPA PCR products was performed on an ABI3500 Genetic Analyzer using the GeneMapper software (Applied Biosystems, Foster City, Calif., USA). For copy number analysis, the data generated was intranormalized by dividing the peak area of each amplification product by the total area of the reference probes only. The ratios were then obtained by dividing the intranormalized probe ratio in a sample by the average intranormalized probe ratio of all reference runs. For methylation analysis, the intranormalized peak area of each MS-MLPA probe from the digested sample was divided by the value obtained for the undigested sample.

\section{Methylation Status Confirmation by Pyrosequencing}

In order to quantify and confirm the results obtained by MSMLPA, pyrosequencing assays were performed. First, $500 \mathrm{ng}$ DNA was subjected to sodium bisulfite treatment and purified using the EZ GOLD methylation kit (ZYMO, Irvine, Calif., USA) according to the manufacturer's instructions. KvDMR promoter PCR amplification was performed in a $25-\mu$ l reaction containing
HotStarTaq Master Mix (Qiagen), $0.1 \mu \mathrm{M}$ forward primer KvDMR-F, 5'-TGTTTAGGTTAGGTTGTATTGTTG-3', and biotinylated reverse primer KvDMR-R, $5^{\prime}$-CCCCATCTCTCTAAAAAAATTT- $3^{\prime}$ (the reverse primer was $5^{\prime}$-biotinylated to facilitate single-strand DNA template isolation for the pyrosequencing reaction). The amplification was carried out according to the routine protocol: denaturation at $95^{\circ} \mathrm{C}$ for $10 \mathrm{~min}$, followed by 45 cycles at $95^{\circ} \mathrm{C}$ for $30 \mathrm{~s}, 56^{\circ} \mathrm{C}$ for $30 \mathrm{~s}, 72^{\circ} \mathrm{C}$ for $30 \mathrm{~s}$, and a final extension at $72^{\circ} \mathrm{C}$ for $7 \mathrm{~min}$. The entire biotinylated PCR product was mixed with $40 \mu \mathrm{l}$ of binding buffer and $2 \mu \mathrm{l}(10 \mathrm{mg} / \mathrm{ml})$ streptavidin-coated polystyrene beads. Bead-amplicon complexes were captured on a vacuum prep tool (Qiagen), and the PCR products were denatured using $0.2 \mathrm{M} \mathrm{NaOH}$. The denatured DNA was resuspended in $0.3 \mu \mathrm{M}$ of sequencing primer $\left(5^{\prime}\right.$-GGGTATATAGTTTATTTTAGTA- $3^{\prime}$ ) dissolved in annealing buffer, and primer annealing was achieved by heating the sample to $80^{\circ} \mathrm{C}$ for $2 \mathrm{~min}$ before cooling to room temperature. The pyrosequencing reaction was carried out on a PyroMark Q24 instrument (Qiagen). The PyroMark Q24 v2.0.6.20 software automatically determines individual methylation frequencies for all $\mathrm{CpG}$ sites in the amplicon; the degree of methylation is calculated from the ratio of the peak heights of $\mathrm{C}$ and $\mathrm{T}$. 
Table 1. KvDMR methylation in different tissues of twins clinically discordant for BWS

\begin{tabular}{lllll}
\hline \multirow{2}{*}{ Region } & Tissue & \multicolumn{2}{l}{ Methylation status } & \multirow{2}{*}{ Reference } \\
\cline { 3 - 4 } & & affected twin & unaffected twin & \\
\hline \multirow{2}{*}{ KvDMR } & fibroblasts & 0.20 & 0.55 & Weksberg et al., 2002 \\
& lymphoblasts & 0.32 & 0.45 & \multirow{2}{*}{ Bliek et al., 2009 } \\
\hline KCNQ1OT1 & blood & 0.20 & normal & Tierling et al., 2011 \\
& blood & 0.16 & 0.22 & \\
\hline IGF2 & fibroblasts & 0.13 & 0.40 & \\
\hline KvDMR & buccal swab & 0.25 & 0.45 & Tierling et al., 2011 \\
& saliva & 0.16 & 0.42 & this study (MS-MLPA/ \\
& fibroblasts & 0.16 & 0.30 & pyrosequencing) \\
\hline
\end{tabular}

Table 2. Microsatellite typing results at 7 different chromosomes confirming monozygosity of the twins

\begin{tabular}{llllll}
\hline Marker & \multicolumn{2}{l}{ Index patient } & & \multicolumn{2}{l}{ Twin } \\
\cline { 2 - 3 } \cline { 5 - 5 } & allele 1 & allele 2 & & allele 1 & allele 2 \\
\hline D3S3694 & 134 & 149 & & 134 & 149 \\
D7S2519 & 111 & 117 & & 111 & 117 \\
D8S277 & 156 & 177 & & 156 & 177 \\
D13S1283 & 152 & 168 & & 152 & 168 \\
D16S3276 & 174 & 174 & 174 & 174 \\
D20S171 & 126 & 126 & 126 & 126 \\
D21S2055 & 152 & 197 & 152 & 197 \\
\hline
\end{tabular}

Table 3. Microsatellite typing results at 11p15.5p15.4 discarding paternal UPD

\begin{tabular}{|c|c|c|c|c|c|c|}
\hline \multirow[t]{2}{*}{ Marker } & \multicolumn{2}{|c|}{ Index patient/twin } & \multicolumn{2}{|l|}{ Father } & \multicolumn{2}{|l|}{ Mother } \\
\hline & allele 1 & allele 2 & allele 1 & allele 2 & allele 1 & allele 2 \\
\hline D11S1318 & 126 & 136 & 126 & 126 & 136 & 136 \\
\hline D11S1363 & 244 & 244 & 244 & 250 & 244 & 250 \\
\hline D11S1984 & 191 & 195 & 183 & 191 & 175 & 195 \\
\hline D11S4046 & 194 & 185 & 185 & 194 & 185 & 185 \\
\hline D11S1088 & 214 & 229 & 184 & 214 & 208 & 229 \\
\hline D11S4181 & 207 & 205 & 207 & 207 & 205 & 213 \\
\hline
\end{tabular}

\section{Zygosity Testing}

As no data on placentation was available, zygosity testing was performed on DNA isolated from blood lymphocytes using 13 polymorphic microsatellite markers (D3S3694, D7S2519, D8S277, D11S1318, D11S1363, D11S1984, D11S4046, D11S1088, D11S4181, D13S1283, D16S3276, D20S171, and D21S2055). Mi- crosatellite typing was performed by fluorescent PCR. The results were analyzed with an ABI3500 Genetic Analyzer and Gene Mapper v.4.1 software, using Gene Scan ROX500 as an internal size standard (all from Applied Biosystems).

\section{Results}

The MS-MLPA study of the $11 \mathrm{p} 15$ region revealed hypomethylation at the maternally imprinted KvDMR in all the tissues analyzed for the affected twin, confirming the clinical diagnosis of BWS. Loss of methylation at KvDMR was also found in blood of the unaffected twin. However, when buccal swab was analyzed, a normal methylation pattern was observed. No copy number variation was identified in the tested probes for any of the samples (fig. 1a, b). These results were confirmed and quantified by pyrosequencing (fig. 1c; table 1; online suppl. fig. 1, 2; see www.karger.com/doi/10.1159/000356689 for all online suppl. material), refining that the healthy twin's methylation status was normal.

Both parents presented a normal methylation pattern in all the analyzed tissues.

Microsatellite analysis revealed monozygotic twinning (table 2) and discarded paternal disomy (table 3).

\section{Discussion}

MZ twins result from fecundation of a single ovum by 1 sperm. MZ twins originate from 1 zygote and are considered to be genetically identical. The prevalence of twin births varies between populations [Zwijnenburg et al.,
44

Mol Syndromol 2014;5:41-46 DOI: $10.1159 / 000356689$
Elalaoui/Garin/Sefiani/

Perez de Nanclares 
2010]. The highest prevalence of twinning is reported in Nigeria, where 1 in 12 persons is a member of a twin pair; the lowest twinning rates are found in Asia $(8 / 1,000$ births in China) [Gan et al., 2007; Hoekstra et al., 2008]. In Europe and the USA, the incidence of twinning is 1 twin pair in every 60 births [Hall, 2003; Hoekstra et al., 2008]. The rate of MZ twinning is constant at 3-4 per 1,000 maternities around the world [Tong et al., 1997; Hall, 2003]. The variation in twinning rates is generally accepted as being the result of variation in dizygotic (DZ) twinning rates.

The causes of MZ twinning in humans are unknown. Shur [2009] assumed that (epi)genetic mechanisms may be involved in the phenomenon of splitting of the zygote. Recently, an increasing frequency of discordant MZ twins has been reported [Zwijnenburg et al., 2010]. Various etiologies were suggested, such as chromosomal mosaicism, point mutations, epigenetic phenomena, and many others.

A large number of BWS twins have been described since the first report in 1980 [Berry et al., 1980; Brown, 1986; Litz et al., 1988; Olney et al., 1988; Chien et al., 1990; Franceschini et al., 1993; Leonard and Johnson, 1993; Leonard et al., 1996; Machin, 1996]. Gaston et al. [2001] reported 5 multiple births: 2 pairs of MZ female twins, and $3 \mathrm{DZ}$ twin pairs. The $2 \mathrm{MZ}$ twin pairs both showed hypomethylation at KvDMR. A cohort of 250 BWS patients was described by Weksberg et al. [2002], the frequency of MZ twinning was $8 \%$ as compared with $1 \% \mathrm{DZ}$ twinning. All MZ twins were phenotypically discordant, and hypomethylation of $\mathrm{KvDMR}$ was reported as the cause of BWS. Bliek et al. [2009] reported a cohort of 13 twin pairs (12 twin pairs and 1 triplet). For 11 of the 13 cases, the genetic defect was hypomethylation of the maternal KvDMR. In 1 case, a mosaic trisomy of chromosome $11 \mathrm{p}$ was found to result in an increased methylation level of H19DMR and a decreased methylation level of KvDMR. In the last case, there was no detectable genetic defect. Other genetic defects have been found in BWS twins: uniparental disomy in a male MZ twin pair and a mosaic trisomy $11 \mathrm{p} 15$ in a female $\mathrm{MZ}$ twin pair [Li et al., 2001; Marcus-Soekarman et al., 2004; Smith et al., 2006].
Based on these data, we could summarize that the majority of BWS twins are MZ discordant, and most of them present hypomethylation of KvDMR. The excess of MZ twins among BWS patients with KvDMR hypomethylation suggests that the methylation defect and the twinning process are correlated [Bestor, 2003].

In most of the reports, the MZ twins are females: 12 of the 13 in the paper of Bliek et al. [2009], 8 of the 10 twin pairs reported by Weksberg et al. [2002], with very few cases of male discordant twins [Weksberg et al., 2002; Smith et al., 2006; Bliek et al., 2009; Tierling et al., 2011]. The striking excess of female twins suggests a possible mechanistic link with $\mathrm{X}$ chromosome inactivation [Orstavik et al., 1995].

Here, we report a male MZ twin pair with loss of methylation at KvDMR found in blood of the affected and unaffected twin. However, when buccal swab was analyzed, only the affected twin presented loss of methylation. Recently, Tierling et al. [2011] reported a further case of male MZ twins discordant for BWS. KvDMR hypomethylation was detected in the affected twin in all tissues, whereas for the unaffected twin, KvDMR hypomethylation occurred only in the blood and was not detected in DNA obtained from fibroblasts, saliva and buccal swabs (table 1). Values for methylation defects in swab samples were less marked, both in our patient and in the one reported by Tierling et al. Through this observation, we would like to highlight the importance of the analysis of independent tissues for clinically discordant MZ twins (or any kind of multiplex). The follow-up of our discordant BWS MZ twins will provide more clinical data (because of their short age actually) and disclose other manifestations that are not present as yet in the affected twin.

\section{Acknowledgements}

We thank the patients and their family. The authors thank Valeria Romanelli for technical assistance on KvDMR pyrosequencing. This work was supported by FIS-program (I3SNSCA10/01056 to I.G.); I3SNS Program of the Spanish Ministry of Health (CP03/0064; SIVI 1395/09 to G.P.N.), and Fundacion Eugenio Rodriguez Pascual to G.P.N.

\section{References}

\footnotetext{
Baujat G, Rio M, Rossignol S, Sanlaville D, Lyonnet $\mathrm{S}$, et al: Paradoxical NSD1 mutations in Beckwith-Wiedemann syndrome and 11p15 anomalies in Sotos syndrome. Am J Hum Genet 74:715-720 (2004).

Beckwith JB: Extreme cytomegaly of the adrenal fetal cortex, omphalocele, hyperplasia of kid-
}

neys and pancreas, and Leydig-cell hyperplasia: another syndrome? Abstract, Western Society for Pediatric Research (1963).

Berry AC, Belton EM, Chantler C: Monozygotic twins discordant for Wiedemann-Beckwith syndrome and the implications for genetic counselling. J Med Genet 17:136-138 (1980).
Monozygotic Male Twin Pair Discordant for BWS
Mol Syndromol 2014;5:41-46 DOI: $10.1159 / 000356689$ 
Bestor TH: Imprinting errors and developmental asymmetry. Philos Trans R Soc Lond B Biol Sci 358:1411-1415 (2003).

- Bliek J, Maas SM, Ruijter JM, Hennekam RC, Alders $\mathrm{M}$, et al: Increased tumour risk for BWS patients correlates with aberrant $\mathrm{H} 19$ and not KCNQ1OT1 methylation: occurrence of KCNQ1OT1 hypomethylation in familial cases of BWS. Hum Mol Genet 10:467-476 (2001).

Bliek J, Alders M, Maas SM, Oostra RJ, Mackay DM, et al: Lessons from BWS twins: complex maternal and paternal hypomethylation and a common source of haematopoietic stem cells. Eur J Hum Genet 12:1625-1634 (2009).

Brown S: Wiedemann-Beckwith syndrome in one of monozygotic twins. Arch Dis Child 6:717 (1986).

-Chien CH, Lee JS, Tsai WY, Wang TR: Wiedemann-Beckwith syndrome with congenital central hypothyroidism in one of monozygotic twins. J Formos Med Assoc 89:132-136 (1990).

- Cooper WN, Luharia A, Evans GA, Raza H, Haire AC, et al: Molecular subtypes and phenotypic expression of Beckwith-Wiedemann syndrome. Eur J Hum Genet 13:1025-1032 (2005).

- Elliott M, Bayly R, Cole T, Temple IK, Maher ER: Clinical features and natural history of Beckwith-Wiedemann syndrome: presentation of 74 new cases. Clin Genet 46:168-174 (1994).

Engstrom W, Lindham S, Schofield P: Wiedemann-Beckwith syndrome. Eur J Pediatr 147: 450-457 (1988).

-Franceschini P, Guala A, Vardeu MP, Franceschini D: Monozygotic twinning and Wiedemann-Beckwith syndrome. Am J Med Genet 46:353-354 (1993).

Gan JP, Wu ZH, Tu ZM, Zheng J: The comparison of twinning rates between urban and rural areas in China. Twin Res Hum Genet 10:633637 (2007).

Gaston V, Le Bouc Y, Soupre V, Burglen L, Donadieu J, et al: Analysis of the methylation status of the KCNQ1OT and H19 genes in leukocyte DNA for the diagnosis and prognosis of Beckwith-Wiedemann syndrome. Eur J Hum Genet 9:409-418 (2001).
Hall JG: Twinning. Lancet 362:735-743 (2003).

Hoekstra C, Zhao ZZ, Lambalk CB, Willemsen G, Martin NG, et al: Dizygotic twinning. Hum Reprod Update 14:37-47 (2008).

Lapunzina P: Risk of tumorigenesis in overgrowth syndromes: a comprehensive review. Am J Med Genet 137:53-71 (2005).

Leonard NJ, Johnson CL: Male monozygotic twins discordant for Beckwith-Wiedemann syndrome. Am J Hum Genet 53:A466 (1993).

Leonard NJ, Bernier FP, Rudd N, Machin GA, Bamforth F, et al: Two pairs of male monozygotic twins discordant for Beckwith-Wiedemann syndrome. Am J Med Genet 61:253257 (1996).

Li M, Squire J, Shuman C, Fei YL, Atkin J, et al: Imprinting status of 11 p15 genes in Beckwith-Wiedemann syndrome patients with CDKN1C mutations. Genomics 74:370-376 (2001).

Litz CE, Taylor KA, Qiu JS, Pescovitz OH, de Martinville B: Absence of detectable chromosomal and molecular abnormalities in monozygotic twins discordant for the WiedemannBeckwith syndrome. Am J Med Genet 30: 821-833 (1988).

Machin GA: Some causes of genotypic and phenotypic discordance in monozygotic twin pairs. Am J Med Genet 61:216-228 (1996).

Marcus-Soekarman D, Hamers G, Velzeboer S, Nijhuis J, Loneus WH, et al: Mosaic trisomy $11 \mathrm{p}$ in monozygotic twins with discordant clinical phenotypes. Am J Med Genet A 124A:288-291 (2004).

- Meyer E, Lim D, Pasha S, Tee LJ, Rahman F, et al: Germline mutation in NLRP2 (NALP2) in a familial imprinting disorder (Beckwith-Wiedemann syndrome). PLoS Genet 3:e1000423 (2009).

Olney AH, Buehler BA, Waziri M: WiedemannBeckwith syndrome in apparently discordant monozygotic twins. Am J Med Genet 29:491499 (1988).

Orstavik RE, Tommerup N, Eiklid K, Orstavik $\mathrm{KH}$ : Non-random X chromosome inactivation in an affected twin in a monozygotic twin pair discordant for Wiedemann-Beckwith syndrome. Am J Med Genet 56:210-214 (1995).

- Pettenati MJ, Haines JL, Higgins RR, Wappner RS, Palmer CG, Weaver DD: WiedemannBeckwith syndrome: presentation of clinical and cytogenetic data on 22 new cases and review of the literature. Hum Genet 74:143-154 (1986).
Reik W, Maher ER: Imprinting in clusters: lessons from Beckwith-Wiedemann syndrome. Trends Genet 13:330-334 (1997).

Shur N: The genetics of twinning: from splitting eggs to breaking paradigms. Am J Med Genet Part C Semin Med Genet 151C:105-109 (2009).

Smith AC, Rubin T, Shuman C, Estabrooks L, Aylsworth AS, et al: New chromosome 11p15 epigenotypes identified in male monozygotic twins with Beckwith-Wiedemann syndrome. Cytogenet Genome Res 113:313-317 (2006).

Tan TY, Amor DJ: Tumour surveillance in Beckwith-Wiedemann syndrome and hemihyperplasia: a critical review of the evidence and suggested guidelines for local practice. J Paediatr Child Health 42:486-490 (2006).

- Tierling S, Souren NY, Reither S, Zang KD, MengHentschel J, et al: DNA methylation studies on imprinted loci in a male monozygotic twin pair discordant for Beckwith-Wiedemann syndrome. Clin Genet 79:546-553 (2011).

Tong S, Caddy D, Short RV: Use of dizygotic to monozygotic twinning ratio as a measure of fertility. Lancet 349:843-845 (1997).

-Weksberg R, Shuman C, Caluseriu O, Smith AC, Fei YL, et al: Discordant KCNQ1OT1 imprinting in sets of monozygotic twins discordant for Beckwith-Wiedemann syndrome. Hum Mol Genet 11:1317-1325 (2002).

Weksberg R, Shuman C, Smith AC: BeckwithWiedemann syndrome. Am J Med Genet C Semin Med Genet 137C:12-23 (2005).

Weksberg R, Shuman C, Beckwith JB: BeckwithWiedemann syndrome. Eur J Hum Genet 18: 8-14 (2010).

Weng EY, Moeschler JB, Graham JM Jr: Longitudinal observations on 15 children with Wiedemann-Beckwith syndrome. Am J Med Genet 56:366-373 (1995).

Wiedemann HR: Complexe malformatif familial avec hernie ombilicale et macroglossie, un 'syndrome nouveau'. J Genet Hum 13:223232 (1964)

Zwijnenburg PJ, Meijers-Heijboer H, Boomsma DI: Identical but not the same: the value of discordant monozygotic twins in genetic research. Am J Med Genet B Neuropsychiatr Genet 153:1134-1149 (2010). 